\title{
AN APPROACH TO MASSIVE MULTICHANNEL BROADBAND FEEDFORWARD ACTIVE NOISE CONTROL USING WAVE-DOMAIN ADAPTIVE FILTERING
}

\author{
Sascha Spors and Herbert Buchner \\ Deutsche Telekom Laboratories, Berlin University of Technology, \\ Ernst-Reuter-Platz 7, 10587 Berlin, Germany \\ \{sascha.spors, herbert.buchner\}@telekom.de
}

\begin{abstract}
Multichannel active noise control (ANC) systems are increasingly being applied in scenarios where an enlarged quiet zone is desired. For few channels numerous solutions to this problem have been developed in the past. However, algorithms for multichannel ANC with a high number of channels (massive multichannel ANC), in order to achieve a large quiet zone, still remain a challenge. The fundamental limitations of current adaptation algorithms in the context of massive multichannel ANC are outlined in this contribution. As a solution to these limitations, the application of the generic concept of wave-domain adaptive filtering (WDAF) is proposed for ANC. Simulation results from a 60-channel ANC system illustrate the successful application of the proposed concepts.
\end{abstract}

\section{INTRODUCTION}

Acoustic ANC aims at suppressing an undesired noise based on the principle of superposition using appropriately driven loudspeakers. The loudspeaker driving signals are typically derived from acoustic measurements and adaptive algorithms in order to cope with arbitrary noise fields and the time varying nature of acoustics. For some applications a large zone where the noise is canceled (quiet zone) is desirable. However, a large quiet zone requires a high number of reproduction and analysis channels. Current multichannel adaptation algorithms are subject to fundamental limitations for such massive multichannel scenarios. This paper will present an advanced adaptation scheme that is capable to overcome the limitations of traditional adaptation algorithms. The next subsection introduces the multichannel broadband feedforward ANC scenario considered throughout this paper.

\subsection{Active Noise Control}

The discrete time and space block diagram shown in Fig. 1 illustrates a generic multichannel ANC system without feedback paths, i.e. we assume that the effects of feedback from the loudspeakers to the reference microphones can be ignored. The matrices of impulse responses $\mathbf{P}(k), \mathbf{S}(k)$ and $\mathbf{C}(k)$ characterize the primary paths between the reference microphones and the error microphones, the secondary paths between the loudspeakers and the error microphones, and the compensation filters used to generate the loudspeaker signals from the reference signals. The discrete time index is denoted by $k$. The elements of the matrices are composed from the k-th element of the impulse response from one input to one output. The signals of the reference microphones are combined in the vector $\mathbf{x}^{(\mathrm{R})}(k)=\left[x_{1}(k), x_{2}(k), \cdots, x_{R}(k)\right]^{T}$,

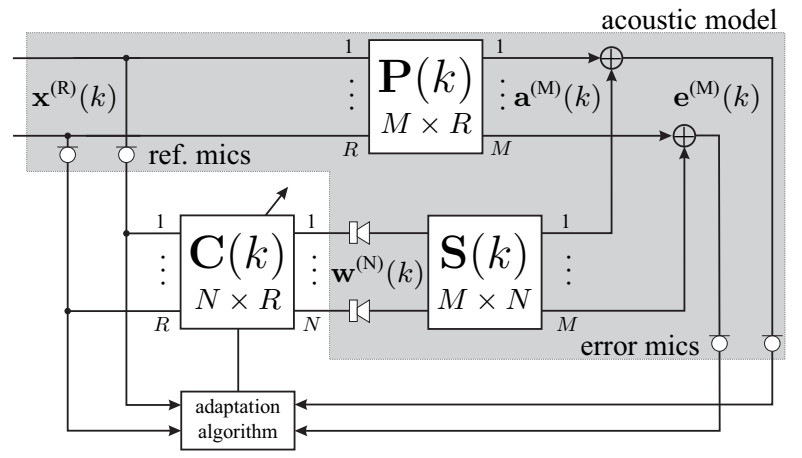

Figure 1: Block diagram illustrating the generic feedforward broadband multichannel ANC system without feedback paths.

where $x_{r}(k)$ denotes the signal of the $r$-th microphone. The signals from the error microphones are denoted by the vector $\mathbf{e}^{(\mathrm{M})}(k)$, the loudspeaker driving signals by $\mathbf{w}^{(\mathrm{N})}(k)$ and the noise field at the error microphones (without ANC) by $\mathbf{a}^{(\mathrm{M})}(k)$.

The reference signal is filtered by the compensation filters and reproduced by the loudspeakers in order to archive the desired goal of a quiet zone. The primary path response $\mathbf{P}(k)$ and secondary path response $\mathbf{S}(k)$ are in general not known a-priori and may change over time due to a varying acoustic environment. This calls for an adaptive computation of the compensation filters. The fundamental problem of adaptive ANC is to compute compensation filters $\mathbf{C}(k)$ on the basis of the reference $\mathbf{x}^{(\mathrm{R})}(k)$ and error signals $\mathbf{e}^{(\mathrm{M})}(k)$ such that the error signal is minimized w.r.t. a given cost function. This problem can be interpreted as a multipleinput/multiple-output (MIMO) pre-equalization problem. Frequencydomain descriptions are derived by transforming each element of the respective time-domain vectors and matrices with a discrete time Fourier transformation (DTFT). Frequency domain quantities are underlined, the temporal frequency is denoted by $\omega$.

\subsection{Traditional Adaptation of Compensation Filters}

We now briefly review the optimum least-squares solution for the filter coefficients, i.e. the derivation of the normal equation for the ANC framework introduced in Section 1.1. The normal equation is the basis for various adaptation algorithms, like the filtered-x recursive least squares algorithm (X-RLS). A detailed discussion can be found, e. g. , in $[1,2]$. 
The least-squares error (LSE) cost function is given as

$$
\xi(\hat{\mathbf{c}}, k)=\sum_{\kappa=0}^{k} \lambda^{k-\kappa} \sum_{m=1}^{M}\left|e_{m}(\kappa)\right|^{2},
$$

where $0<\lambda<1$ denotes an exponential weighting factor. The normal equation is derived by expressing the error $\mathbf{e}^{(\mathrm{M})}(k)$ using the filter coefficients, introducing the result into the cost function (1) and calculating its gradient. The optimal filter coefficients in the mean-squared error (MSE) sense are found by setting the gradient of the cost function to zero. The resulting normal equation is given as

$$
\hat{\mathbf{\Phi}}_{x x}(k) \hat{\mathbf{c}}(k)=\hat{\mathbf{\Phi}}_{x a}(k),
$$

where the $N_{c} N R \times 1$ vector $\hat{\mathbf{c}}(k)$ denotes a vector composed from all estimated filter coefficients at time instant $k$ and $N_{c}$ the number of filter coefficients. The $N R N_{c} \times N_{c} R N$ matrix $\hat{\mathbf{\Phi}}_{x x}$ denotes the averaged correlation matrix of the filtered reference signals. The filtered reference signals are defined by the convolution $x_{r}(k) * s_{m, n}(k)$ of the reference signals with the secondary path responses. The $N R N_{c} \times 1$ vector $\hat{\boldsymbol{\Phi}}_{x a}$ denotes the averaged correlation vector between these filtered reference signals and the noise field at the error sensors (without ANC). The optimal compensation filter w.r.t. the cost function (1) is given by solving the normal equation (2). The X-RLS algorithm can be derived from the normal equation (2) by computing $\hat{\boldsymbol{\Phi}}_{x x}(k)$ and $\hat{\boldsymbol{\Phi}}_{x a}(k)$ in a recursive fashion and applying the matrix inversion lemma [1]. The calculation of the filtered reference signals requires knowledge of the secondary path response $\mathbf{S}(k)$, which is in general not known a-priori and may be time-variant. Hence, the secondary path characteristics have to be identified additionally in a practical system.

\section{MASSIVE MULTICHANNEL ANC}

In order to derive an ANC system with enlarged quiet zone, massive multichannel ANC will be introduced in the following.

\subsection{Basic Concept}

The solution of the homogeneous wave equation in the (temporal) frequency domain for a bounded region $V$ w.r.t. inhomogeneous boundary conditions is given by the Kirchhoff-Helmholtz integral [3]

$$
\begin{aligned}
\underline{p}(\mathbf{x}, \omega)=-\oint_{\partial V}\left\{\underline{g}\left(\mathbf{x} \mid \mathbf{x}_{0}, \omega\right) \frac{\partial}{\partial \mathbf{n}} \underline{p}\left(\mathbf{x}_{0}, \omega\right)-\right. \\
\left.-\underline{p}\left(\mathbf{x}_{0}, \omega\right) \frac{\partial}{\partial \mathbf{n}} \underline{g}\left(\mathbf{x} \mid \mathbf{x}_{0}, \omega\right)\right\} d S_{0},
\end{aligned}
$$

where $\underline{p}(\mathbf{x}, \omega)$ denotes the pressure field inside a bounded region $V$ surrounded by the border $\partial V(\mathbf{x} \in V), \underline{g}\left(\mathbf{x} \mid \mathbf{x}_{0}, \omega\right)$ the free-field Green's function, $\underline{p}\left(\mathbf{x}_{0}, \omega\right)$ the acoustic pressure at the boundary $\partial V\left(\mathbf{x}_{0} \in \partial V\right)$ and $\frac{\partial}{\partial \mathbf{n}}$ the directional gradient in direction of the inward pointing normal vector $\mathbf{n}$ of $V$. It should be noted for the following that the free-field Green's function represents the wave field of a monopole source placed at the point $\mathbf{x}_{0}$ and its directional gradient the field of a dipole source.

Eq. (3) can be interpreted in two ways: (1) if the acoustic pressure and its directional gradient are known at the boundary $\partial V$ then the acoustic pressure inside $V$ can be calculated and (2) if a

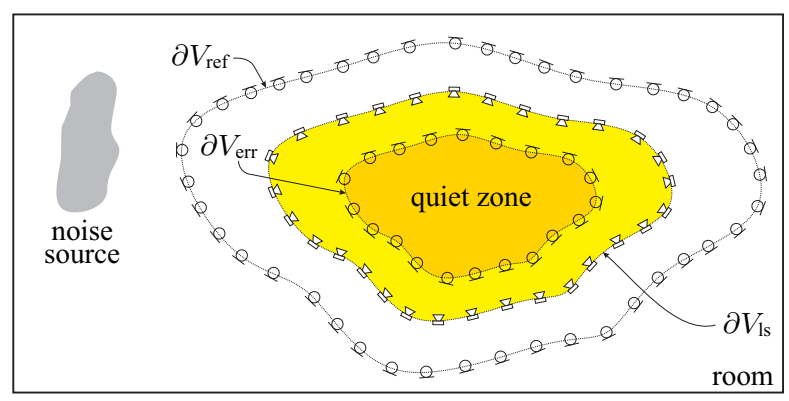

Figure 2: Massive multichannel ANC with enlarged quite zone based on the Kirchhoff-Helmholtz integral.

continuous distribution of monopole and dipole sources is placed on the border and driven properly then the wave field in $V$ can be fully controlled. The first interpretation is the basis of wave field analysis (WFA), while the second is the basis of many sound reproduction systems like, e.g., wave field synthesis (WFS). A suitable combination of both principles will result in an enlarged quiet zone for broadband feedforward ANC as will be shown.

Figure 2 illustrates the setup considered in the following. A distributed noise source located outside the ANC system emits noise that is scattered at the walls of a reverberant room. The resulting complex noise field and its residual (after compensation) is analyzed by reference microphones and error microphones arranged on the closed contours $\partial V_{\text {ref }}$ and $\partial V_{\text {err }}$. The wave field is controlled by the loudspeakers arranged on the contour $\partial V_{\mathrm{ls}}$. For continuous microphone and loudspeaker distributions a perfect analysis and control of the wave field is possible according to Eq. (3). The result will be an enlarged quiet zone which fully covers $V_{\text {err. }}$.

For a practical implementation of the proposed ANC scheme spatial sampling of the continuous microphone and loudspeaker distributions is required. As a consequence, active compensation of the noise field is only possible for a limited bandwidth. For a reasonable bandwidth and size of the quiet zone a high number of microphones and loudspeakers is required. This results in a massive multichannel adaptation problem and the fundamental problems outlined in Section 3.1. A solution to these will be provided in Section 3.2 and Section 3.3 by eigenspace considerations and the WDAF concept. We limit ourselves to two-dimensional wave fields for the following discussion of WFS and WFA. An extension to the three-dimensional case is straightforward.

\subsection{Wave Field Synthesis}

WFS is typically a two-dimensional spatial sound reproduction technique which is based on the Kirchhoff-Helmholtz integral [4]. Several simplifications are necessary to arrive at a realizable reproduction system. One of the simplifications is to neglect the dipole secondary sources dictated by the Kirchhoff-Helmholtz integral (3). WFS uses only loudspeakers with closed cabinets as approximation of the required monopole sources. A consequence of the applied approximations are several reproduction artifacts. Their influence on ANC have been investigated in the previous study [5].

\subsection{Wave Field Analysis}

For a wide variety of applications it is convenient to represent acoustic wave fields w.r.t. an orthogonal basis. A suitable basis 
will depend on the underlying coordinate system. The representations of a wave field that are connected to Cartesian and polar coordinates decompose a wave field into plane waves and circular harmonics, respectively [3]. Of special interest within the scope of this paper is the decomposition of an acoustic wave field into circular harmonics. This decomposition is given as follows [3]

$$
\begin{aligned}
& \underline{p}(\alpha, r, \omega)= \\
& \sum_{\nu=-\infty}^{\infty} \underline{\breve{p}}^{(1)}(\nu, \omega) H_{\nu}^{(1)}\left(\frac{\omega}{c} r\right) e^{j \nu \alpha}+\underline{\breve{p}}^{(2)}(\nu, \omega) H_{\nu}^{(2)}\left(\frac{\omega}{c} r\right) e^{j \nu \alpha}
\end{aligned}
$$

where $\breve{p}^{(1),(2)}(\nu, \omega)$ denote the circular harmonics expansion coefficients, $H_{\nu}^{(1),(2)}$ the Hankel function of $\nu$-th order of first/second kind, $\nu$ the angular frequency, $r$ and $\alpha$ the radial and angular coordinate of the polar coordinate system and $c$ the speed of sound. It can be shown that $\underline{\breve{p}}^{(1)}(\nu, \omega)$ represents the expansion of an incoming wave field w.r.t. the origin and $\underline{\breve{p}}^{(2)}(\nu, \omega)$ the expansion of an outgoing wave field [3].

The Kirchhoff-Helmholtz integral states that it is possible to measure an arbitrary wave field on the boundary of a bounded region to derive the circular harmonics expansion coefficients. Due to the underlying geometry circular microphone arrays are well suited for this task. Suitable techniques can be found in [6].

\section{EFFICIENT ADAPTATION FOR MASSIVE MULTICHANNEL ANC}

\subsection{Fundamental Problems of Traditional Filter Adaptation}

It can be concluded from the normal equation (2) that the adaptation of the compensation filters for the massive multichannel case is subject to fundamental problems:

1. Ill-conditioning of the correlation matrix $\hat{\mathbf{\Phi}}_{x x}(k)$, and

2. computational complexity for massive multichannel ANC.

Both problems are related to the solution of the normal Eq. (2) by the adaptive algorithm. The correlation matrix $\hat{\boldsymbol{\Phi}}_{x x}(k)$ expresses the auto- and cross-correlations of the filtered reference signals. Due to linear relations between the channels, and hence spatiotemporal correlations, $\hat{\boldsymbol{\Phi}}_{x x}(k)$ will generally be ill-conditioned. In the extreme case, depending on the reference signals $\mathbf{x}^{(\mathrm{R})}(k)$, there may be multiple possible solutions for $\hat{\mathbf{c}}$ that minimize $\xi(\hat{\mathbf{c}}, k)$.

Due to the dimensionality of the correlation matrix $\hat{\boldsymbol{\Phi}}_{x x}(k)$ the solution of the normal equation may become an infeasible task for massive multichannel ANC systems. The computational complexity of the filter adaptation is enormous even for modest channel numbers [1]. The same problems as outlined above hold also for the identification of the secondary path response.

\subsection{Eigenspace Adaptive Filtering}

Eigenspace adaptive filtering (EAF) provides a generic framework for MIMO pre-equalization which explicitly solves the second problem by utilizing signal and system transformations. The conditioning of $\hat{\boldsymbol{\Phi}}_{x x}(k)$ is also highly alleviated by removing all crosschannel correlations. In the following a brief review of EAF based on [2] will be given. The basic idea is to perform a decoupling of the MIMO systems $\underline{\mathbf{S}}(\omega)$ and $\underline{\mathbf{P}}(\omega)$, resulting in a decoupling of the MIMO adaptation problem and the correlation matrix $\hat{\mathbf{\Phi}}_{x x}(k)$.

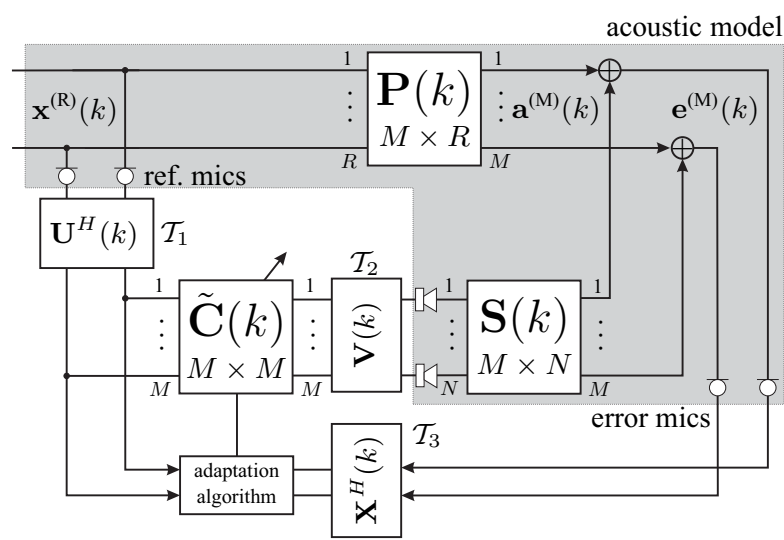

Figure 3: Block diagram illustrating the eigenspace and wavedomain adaptive filtering approach to massive multichannel ANC.

The generalized singular value decomposition (GSVD) [7] will be used to derive the desired decoupling. The GSVD for the matrices $\underline{\mathbf{S}}(\omega)$ and $\underline{\mathbf{P}}(\omega)$ is given as follows:

$$
\begin{aligned}
& \underline{\mathbf{S}}(\omega)=\underline{\mathbf{X}}(\omega) \underline{\tilde{\mathbf{S}}}(\omega) \underline{\mathbf{V}}^{H}(\omega), \\
& \underline{\mathbf{P}}(\omega)=\underline{\mathbf{X}}(\omega) \underline{\tilde{\mathbf{P}}}(\omega) \underline{\mathbf{U}}^{H}(\omega) .
\end{aligned}
$$

The matrices $\underline{\mathbf{X}}(\omega), \underline{\mathbf{V}}(\omega)$ and $\underline{\mathbf{U}}(\omega)$ are unitary matrices. The matrix $\underline{\mathbf{X}}(\omega)$ is the generalized singular matrix of $\underline{\mathbf{S}}(\omega)$ and $\underline{\mathbf{P}}(\omega)$, the matrices $\underline{\mathbf{V}}(\omega)$ and $\underline{\mathbf{U}}(\omega)$ the respective right singular matrices of $\underline{\mathbf{S}}(\omega)$ and $\underline{\mathbf{P}}(\omega)$. The matrices $\underline{\tilde{\mathbf{S}}}(\omega)$ and $\underline{\tilde{\mathbf{P}}}(\omega)$ are diagonal matrices constructed from the singular values of $\underline{\mathbf{S}}(\omega)$ and $\underline{\mathbf{P}}(\omega)$. A decomposition of the compensation filters is derived by expressing the non-adaptive LSE solution $\underline{\mathbf{C}}(\omega)=-\underline{\mathbf{S}}^{+}(\omega) \underline{\mathbf{P}}(\omega)$ using the singular matrices $\left(\underline{\mathbf{S}}^{+}(\omega)\right.$ denotes the pseudoinverse of $\left.\underline{\mathbf{S}}(\omega)\right)$. It is given as [2]

$$
\underline{\mathbf{C}}(\omega)=\underline{\mathbf{V}}(\omega) \underline{\tilde{\mathbf{C}}}(\omega) \underline{\mathbf{U}}^{H}(\omega),
$$

where $\underline{\tilde{\mathbf{C}}}(\omega)$ denotes a diagonal matrix. The error signal $\underline{\mathbf{e}}^{(\mathrm{M})}(\omega)$ at the $M$ analysis positions can be derived as

$$
\underline{\mathbf{e}}^{(\mathrm{M})}(\omega)=\underline{\mathbf{P}}(\omega) \underline{\mathbf{x}}^{(\mathrm{R})}(\omega)+\underline{\mathbf{S}}(\omega) \underline{\mathbf{C}}(\omega) \underline{\mathbf{x}}^{(\mathrm{R})}(\omega) .
$$

Introducing Eq. (5) and Eq. (6) into (7), exploiting the unitary property of the singular matrices and multiplying both sides with $\underline{\mathbf{X}}^{H}(\omega)$ yields

$$
\underline{\tilde{\mathbf{e}}}^{(\mathrm{M})}(\omega)=\underline{\tilde{\mathbf{P}}}(\omega) \underline{\tilde{\mathbf{x}}}^{(\mathrm{M})}(\omega)+\underline{\tilde{\mathbf{S}}}(\omega) \underline{\tilde{\mathbf{C}}}(\omega) \underline{\tilde{\mathbf{x}}}^{(\mathrm{M})}(\omega),
$$

where $\underline{\tilde{\mathbf{e}}}^{(\mathrm{M})}(\omega)=\underline{\mathbf{X}}^{H}(\omega) \underline{\mathbf{e}}^{(\mathrm{M})}(\omega)$ and $\underline{\tilde{\mathbf{x}}}^{(\mathrm{M})}(\omega)=\underline{\mathbf{U}}^{H}(\omega) \underline{\mathbf{x}}^{(\mathrm{R})}(\omega)$ denote the error and reference signal vector in the transformed domain. Equation (8) states that the adaptive inverse MIMO filtering problem is decomposed into $M$ single-channel adaptive inverse filtering problems using the GSVD. The adaptation of the compensation filters is performed independently for each of the transformed components. Introducing the transformations into Fig. 1 yields Fig. 3 which illustrates the application of eigenspace adaptive filtering to ANC. Note that $\underline{\mathbf{w}}^{(\mathrm{N})}(\omega)=\underline{\mathbf{V}}(\omega) \underline{\tilde{\mathbf{w}}}^{(\mathrm{M})}(\omega)$.

Although EAF provides the optimal solution to massive multichannel ANC w.r.t. the desired decoupling it has two major drawbacks: (1) the GSVD is computationally quite complex and (2) the optimal transformations depend on the secondary path response which is potentially time variant. The next section will introduce wave-domain adaptive filtering (WDAF) as practical solution to these problems. 


\subsection{Wave-Domain Adaptive Filtering using Circular Harmonics}

WDAF is based on approximation of the concept of perfect decoupling by explicit consideration of the characteristics of the propagation medium and the geometry of the underlying acoustic problem. The perfect decoupling of the MIMO adaptation problem is given up in favor of generic and fixed transformations $\mathcal{T}_{1}, \mathcal{T}_{2}$ and $\mathcal{T}_{3}$ (see Fig. 3) which are to some degree independent of the acoustic characteristics. Fixed transformations can provide nearly the same favorable properties as the optimal GSVD-based transformations with the benefit of computational efficiency. It has been shown that the circular harmonics decomposition is a good candidate as wave-domain transformation for circular analysis geometries and not too reverberant acoustic environments [2].

\section{RESULTS}

The next section illustrates the application of WDAF to massive multichannel broadband feedforward ANC. For a first proof of concept a numerically simulated environment was used.

\subsection{Simulation Setup}

The simulated setup consists of: (1) one circular microphone array for analysis of the reference field, (2) one for analysis of the error field and (3) a circular loudspeaker array for active control of the field emitted by a noise source. The reference array has a radius of $R_{\text {ref }}=2 \mathrm{~m}$, the loudspeaker array of $R_{\mathrm{ls}}=1.5 \mathrm{~m}$ and the error array of $R_{\text {err }}=0.75 \mathrm{~m}$. All arrays have 60 equiangular spatial sampling points where the microphones and loudspeakers are placed. The simulation included a reverberant room with size $8 \times 8 \times 3 \mathrm{~m}(\mathrm{w} \times 1 \times \mathrm{h})$ and reflection factor $\rho_{\mathrm{pw}}=0.9$ at the side walls. All arrays are placed concentrically in the center of the room. The mirror image method was applied to simulate reflections. A noise source (point source) emitting white noise was placed at an angle of $\alpha=180^{\circ}$ and a distance of $D=3 \mathrm{~m}$ from the center of the arrays. The circular harmonics expansion coefficients where computed from the microphone signals using [6] for transformations $\mathcal{T}_{1}$ and $\mathcal{T}_{3}$. Transformation $\mathcal{T}_{2}$ was realized using Eq. (4). For the adaptation, perfect knowledge of the secondary path was assumed and no noise was added to the microphone signals. All signals where bandlimited to a bandwidth of $850 \mathrm{~Hz}$ in order to avoid spatial aliasing.

\subsection{Results}

The circular harmonics coefficients of the error signals represent the residual wave field within the error microphones. A decomposition into plane waves is more illustrative in our context. The plane wave decomposition can be derived from the circular harmonics decomposition via a Fourier series [6]. Figure 4(a) shows the energy of the plane wave components of the reference and error field after convergence of the compensation filters. The suppression of the noise source over all incidence angles of the plane waves within the quiet zone can be seen clearly. This effectively results in an enlarged quiet zone.

Figure 4(b) illustrates the filter convergence by showing the energy of the error signal for an omnidirectional pickup (superposition of all plane wave components) at the center of the quiet zone. It can be seen clearly that the adaptation of the compensation filters converges fast and stable for this massive multichannel scenario.

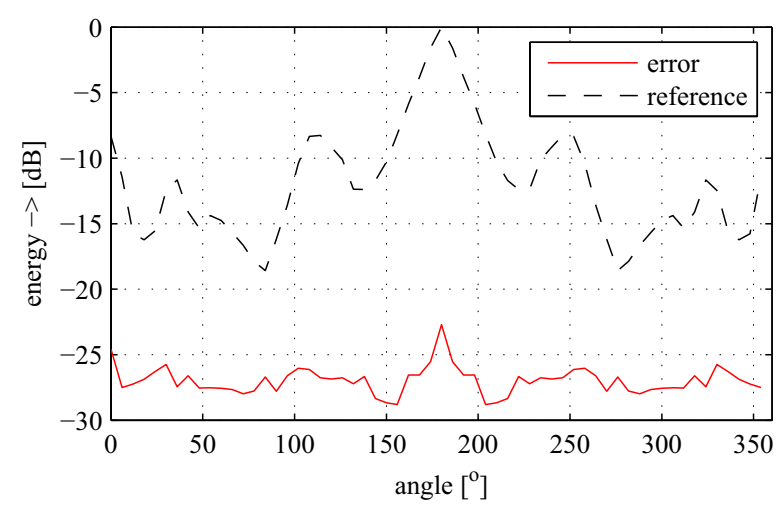

(a) Energy of plane wave components after convergence of the compensation filters.

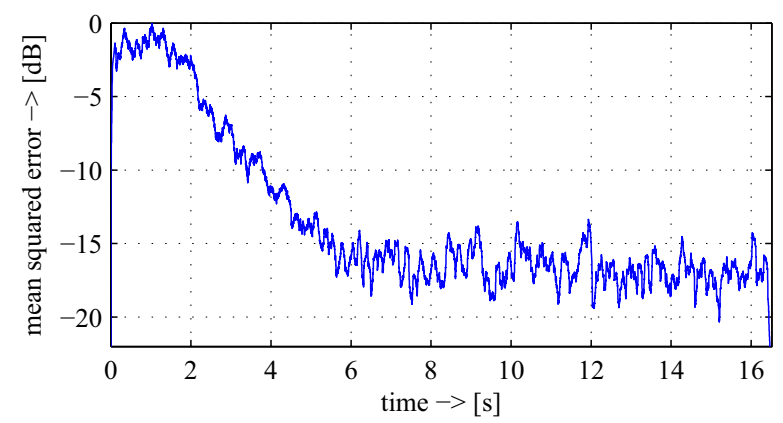

(b) Error signal for omnidirectional pickup at center of array.

Figure 4: Results for simulated massive multichannel ANC system.

\section{CONCLUSIONS}

This paper presents a highly efficient adaptation scheme for massive multichannel ANC systems which is based on WDAF. A decomposition of the MIMO adaptation problem into a series of single channel adaptation problems using signal and system transformations overcomes the fundamental problems of adaptive inverse filtering for massive multichannel systems. The paper presents simulation results as first proof of concept. Further research involves inclusion of the feedback paths into the proposed scheme.

\section{REFERENCES}

[1] M. Bouchard and S. Quednau, "Multichannel recursive-least-squares algorithms and fast-transversal-filter algorithms for active noise control and sound reproduction systems," IEEE Transactions on Speech and Audio Processing, vol. 8, no. 5, pp. 606-618, September 2000.

[2] S. Spors, H. Buchner, and R. Rabenstein, "Eigenspace adaptive filtering for efficient pre-equalization of acoustic MIMO systems," in European Signal Processing Conference, Florence, Italy, Sept. 2006.

[3] E. Williams, Fourier Acoustics: Sound Radiation and Nearfield Acoustical Holography. Academic Press, 1999.

[4] A. Berkhout, "A holographic approach to acoustic control," Journal of the Audio Engineering Society, vol. 36, pp. 977-995, December 1988.

[5] A. Kuntz and R. Rabenstein, "An approach to global noise control by wave field synthesis," in European Signal Processing Conference, Vienna, Sept. 2004.

[6] E. Hulsebos, D. de Vries, and E. Bourdillat, "Improved microphone array configurations for auralization of sound fields by Wave Field Synthesis," in 110th AES Convention. Amsterdam, May 2001.

[7] G. Golub and C. Loan, Matrix Computations. The Johns Hopkins University Press, 1989. 